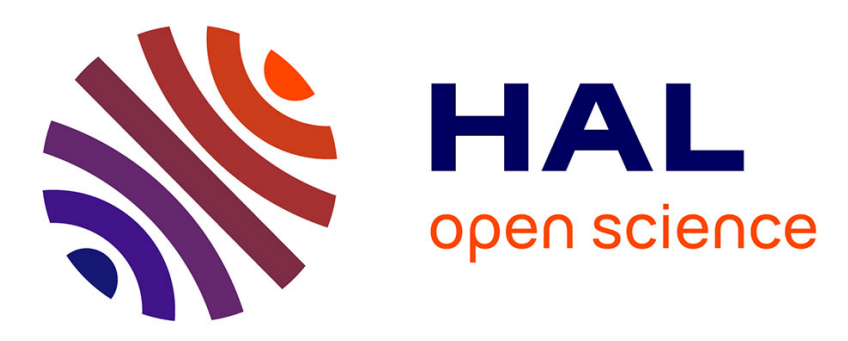

\title{
Molecular genetics of Conn adenomas in the era of exome analysis
}

\author{
Rami El Zein, Sheerazed Boulkroun, Fabio Luiz Fernandes-Rosa, \\ Maria-Christina Zennaro
}

\section{- To cite this version:}

Rami El Zein, Sheerazed Boulkroun, Fabio Luiz Fernandes-Rosa, Maria-Christina Zennaro. Molecular genetics of Conn adenomas in the era of exome analysis. La Presse Médicale, 2018, 47 (7-8), pp.e151e158. 10.1016/j.lpm.2018.07.006 . inserm-03560586

\section{HAL Id: inserm-03560586 https://www.hal.inserm.fr/inserm-03560586}

Submitted on 7 Feb 2022

HAL is a multi-disciplinary open access archive for the deposit and dissemination of scientific research documents, whether they are published or not. The documents may come from teaching and research institutions in France or abroad, or from public or private research centers.
L'archive ouverte pluridisciplinaire HAL, est destinée au dépôt et à la diffusion de documents scientifiques de niveau recherche, publiés ou non, émanant des établissements d'enseignement et de recherche français ou étrangers, des laboratoires publics ou privés. 


\section{Molecular genetics of Conn adenomas in the era of exome analysis}

Rami El Zein ${ }^{1,2}$, Sheerazed Boulkroun ${ }^{1,2}$, Fabio Luiz Fernandes-Rosa ${ }^{1,2,3}$, Maria-Christina Zennaro $^{1,2,3}$

Affiliations:

${ }^{1}$ INSERM, UMRS_970, Paris Cardiovascular Research Center, Paris, France

${ }^{2}$ University Paris Descartes, Sorbonne Paris Cité, Paris, France

${ }^{3}$ Assistance Publique-Hôpitaux de Paris, Hôpital Européen Georges Pompidou, Service de Génétique, Paris, France

Address correspondence to:

Maria-Christina Zennaro

Institut National de la Santé et de la Recherche Médicale, Unité 970

Paris Cardiovascular Research Center - PARCC

56 rue Leblanc

75015 Paris, France

E-mail: maria-christina.zennaro@inserm.fr

Running Title: Genetic basis of primary aldosteronism

Key words: primary aldosteronism, aldosterone producing adenoma, Familial Hyperaldosteronism, somatic mutations, germline mutations, potassium channels, calcium channels, ATPase, wnt/ $\beta$-catenin pathway 


\section{Abstract}

Aldosterone producing adenomas (APA) are a major cause of primary aldosteronism (PA), the most common form of secondary hypertension. Exome analysis of APA has allowed the identification of recurrent somatic mutations in KCNJ5, CACNA1D, ATP1A1, and ATP2B3 in more than $50 \%$ of sporadic cases. These gain of function mutations in ion channels and pumps lead to increased and autonomous aldosterone production. In addition, somatic CTNNB1 mutations have also been identified in APA. The CTNNB1 mutations were also identified in cortisol producing adenomas and adrenal cancer, but their role in APA development and the mechanisms specifying the hormonal production or the malignant phenotype remain unknown. The role of the somatic mutations in the regulation of aldosterone production is well understood, while the impact of these mutations on cell proliferation remains to be established. Furthermore, the sequence of events leading to APA formation is currently the focus of many studies. There is evidence for a two-hit model where the somatic mutations are second hits occurring in a previously remodeled adrenal cortex. On the other hand, the APA-driver mutations were also identified in aldosterone-producing cell clusters (APCC) in normal adrenals, suggesting that these structure may represent precursors for APA development. As PA due to APA can be cured by surgical removal of the affected adrenal gland, the identification of the underlying genetic abnormality by novel biomarkers could improve diagnostic and therapeutic approaches of the disease. In this context, recent data on steroid profiling in peripheral venous samples of APA patients and on new drugs capable of inhibiting mutated potassium channels provide promising preliminary data with potential for translation into clinical care. 


\section{Introduction}

2 Arterial hypertension (HT) is a worldwide health problem which affects $\sim 25 \%$ of the

3 global population [1], resulting in an estimated 9.4 million deaths or approximately $12.8 \%$ of

4 all deaths (Global Health Observatory data, WHO). A vast and diverse array of drugs exists

5 for the treatment of HT, such as diuretics, antagonists of the renin-angiotensin-aldosterone

6 system, notably angiotensin-converting enzyme (ACE) inhibitors and angiotensin receptor

7 blockers, calcium channel blockers, vasodilators, $\beta$ adrenergic blocking agents. Optimal blood

8 pressure control, however, is still far from being achieved in up to two thirds of the

9 hypertensive population. In a certain proportion of cases, HT can arise from a specific

10 disease; endocrine hypertension, a frequent form of secondary arterial hypertension, emerges

11 following a dysregulation of one or more hormones that are involved in blood pressure

12 regulation. Primary aldosteronism (PA), also known as Conn's syndrome, is the most frequent

13 form of secondary hypertension with estimates of up to $10 \%$ of cases in referred patients, $4 \%$

14 in primary care [2] and $20 \%$ in patients with resistant hypertension [3,4]. PA is mainly due to

15 aldosterone producing adenoma (APA) and bilateral adrenal hyperplasias (BAH, or idiopathic

16 hyperaldosteronism, IHA). The clinical picture of patients with PA consists of HT, a high

17 aldosterone to renin ratio, which has become one of the major diagnostic tools for PA

18 alongside different confirmation tests and adrenal venous sampling for subtype diagnosis, and

19 variable hypokalemia and metabolic alkalosis [5]. PA is associated to an increased risk of

20 cardiovascular complications, which occur beyond the effect of hypertension, such as

21 coronary artery disease, heart failure, myocardial infarction, atrial fibrillation and renal

22 damage. Different studies insist on the importance of screening most hypertensive patients for

23 PA to either confirm or exclude the diagnosis [6,7]. Indeed, early PA diagnosis can improve

24 prognosis and prevent the development of target organ damage. 
Although the management of PA in hypertensive patients has come a long way and the treatment is much better established, the prognosis of unilateral PA depends on different criteria such as age, sex, BMI, age upon diagnosis and the duration of hypertension $[7,8]$. Indeed, younger patients and female patients show a better clinical outcome after adrenalectomy in comparison to older or male patients [7].

\section{Aldosterone biosynthesis in the adrenal cortex}

The human adrenal cortex is composed of three distinct zones that are characterized by their respective functions. Steroid hormones are synthesized following the sequential enzymatic breakdown of cholesterol by different cytochrome P450 enzymes as well as hydroxysteroid dehydrogenases, the particularity of each zone lies in the expression of specific steroidogenic enzymes and the ability to have each its own regulators. The most outer zone of the adrenal cortex, the zona glomerulosa ( $\mathrm{ZG})$, expresses aldosterone synthase (encoded by $C Y P 11 B 2$ ), which catalyzes the hydroxylation at the $\mathrm{C} 11$ position of the 11deoxycorticosterone into corticosterone, furthermore, its hydroxylation at $\mathrm{C} 18$ into $18(\mathrm{OH})$ corticosterone followed by an oxidation of C18's hydroxyl group giving as an end result aldosterone. The main trigger for aldosterone biosynthesis is the activation of intracellular calcium signaling in the zona glomerulosa which is induced by either angiotensin II (Ang II) from the renin-angiotensin system or by extracellular potassium levels. The zona fasciculata $(\mathrm{ZF})$ of the adrenal cortex mainly produces cortisol. In this process, the conversion of progesterone into 17-hydroxyprogesterone is catalyzed by the activity of $17 \alpha$-hydroxylase which is not expressed in ZG cells. 17-hydroxyprogesterone undergoes a hydroxylation at 
production is the hypothalamus-pituitary-adrenal (HPA) axis primarily through the adrenocorticotropic hormone (ACTH).

Ang II is one of the major regulators of aldosterone secretion by ZG cells. The binding of AngII to its receptor (AT1) will lead to the activation of the Gaq-phospholipase C-mediated pathway, increasing inositol 1,4,5-triphosphhate (IP3) and 1,2-diacylglycerol concentrations. Ultimately, IP3 is responsible for increased intracellular calcium concentration due to calcium release from intracellular stores. AngII also inhibits the background TWIK-related acidsensitive potassium channel (TASK), as well as GIRK4 and the $\mathrm{Na}+\mathrm{K}+-\mathrm{ATPase}$, leading to a cell membrane depolarization [9]. This will lead to opening of voltage-gated $\mathrm{Ca} 2+$ channels, also increasing intracellular calcium concentrations.

The other main stimulator for aldosterone biosynthesis is the increase in extracellular potassium levels. In the normal ZG cell at a resting state, the cell membrane potential is hyperpolarized, the reason is that the membrane potential follows closely the equilibrium potential of potassium in these cells which largely express potassium channels. Small increases in extracellular potassium levels cause ZG cell membrane depolarization. The depolarization of the ZG cell membrane leads to the opening of voltage gated $\mathrm{Ca}^{2+}$ channels and an increase in intracellular calcium levels resulting in the activation of calcium signaling.

Calcium signaling acts by increasing the release of deesterified cholesterol from cytoplasmic stores, as well as cholesterol delivery to the outer mitochondrial membrane and then to the inner mitochondrial membrane by increasing the expression of the steroid acute regulatory protein (StAR). Calcium signaling also increases the expression of cofactors required for p450 cytochrome enzymes. Calcium/Calmodulin binding in the cytosol of the ZG cell induces the activation of protein kinases that regulate phosphorylation of transcription factors involved in $C Y P 11 B 2$ transcriptional induction, mainly nuclear receptor subfamily 4 
group A 1 and 2 (NR4A1 and NR4A2 coding for NUR77/NGF1B and NURR1 respectively) and the cyclic AMP-responsive element-binding protein (CREB).

\section{Genetic abnormalities in APA}

PA is due to inappropriate aldosterone production by the adrenal cortex in spite of the suppression of the renin-angiotensin system. In the last years, whole exome sequencing (WES) performed on DNA from APA led to the identification of recurrent somatic mutations in genes coding for ion channels (KCNJ5 and CACNA1D) and ATPases (ATP1A1 and ATP2B3). These genes are essential for regulating intracellular ion homeostasis and cell membrane potential. All these mutations promote an increased intracellular calcium signaling through cell membrane depolarization and opening of voltage-dependent calcium channels, or impaired intracellular calcium recycling, therefore leading to high aldosterone levels by constitutive expression of $C Y P 11 B 2$.

In a large multicenter study from the European Network for the Study of Adrenal Tumors (ENS@T) that analyzed somatic mutations in APA from 474 patients [10], hot spot regions for mutations in $K C N J 5, C A C N A 1 D, A T P 1 A 1$ and $A T P 2 B 3$ were sequenced. Somatic mutations were identified in $54.2 \%$ of APA, with $K C N J 5$ being the most prevalent at $38 \%$, $C A C N A 1 D$ at $9.3 \%, A T P 1 A 1$ at $5.3 \%$ and $A T P 2 B 31.7 \%$ of these mutations. However, KCNJ5 mutations are more prevalent in Asian populations, with up to $76 \%$ of prevalence [1115]. These observations were corroborated by a meta-analysis of clinical and genetic data from 1636 patients with APA showing an overall prevalence of $K C N J 5$ mutations of $43 \%$, with higher prevalence in patients from Asia [16]. Some aldosterone producing adenomas also carry somatic mutations in the gene that codes for $\beta$-catenin (CTNNB1), less common mutations have also been identified in PRKACA (encoding Protein Kinase cAMP-Activated Catalytic Subunit $\alpha$ ) [17-19]. 
KCNJ5 codes for an inwardly rectifying $\mathrm{K}^{+}$channel, which is the G-protein-activated inward rectifier potassium channel GIRK4 (also known as Kir3.4). It is mainly expressed in the $\mathrm{ZG}$ of the adrenal cortex. KCNJ5 mutations were found to be more frequent in female and younger patients, and the expression of GIRK4 in APA was found to be correlated to the mutation status [20]. GIRK4 is composed of 2 membrane spanning helices with one pore-

103 forming region in between and $\mathrm{N}$ - and $\mathrm{C}$ - termini that contribute to the pore structure [21].

104 Choi et al identified two somatic KCNJ5 mutations mapping to the selectivity filter of GIRK4 105 (p.Gly151Arg and p.Leu168Arg). In addition to these two mutations (the most prevalent mutations in APA), the majority of the $K C N J 5$ mutations described are located within or near 107 the selectivity filter, rendering the channel permeable to sodium, which leads to chronic cell 108 membrane depolarization [22]. Transient transfection of KCNJ5 mutants in HAC-15 resulted 109 in a calcium-dependent increase in $C Y P 11 B 2$ expression and aldosterone biosynthesis in the 110 cells; the mutant GIRK4, however, did not induce any increase in proliferation but rather a 111 reduced cell viability or sodium-induced cell death $[23,24]$. This leaves the question of the 112 role of KCNJ5 mutations on the cell proliferation and APA formation unanswered in tumors 113 where these mutations occur. KCNJ5 mRNA expression is not affected by KCNJ5 mutations, 114 but APA harboring KCNJ5 mutations show decreased GIRK4 protein expression when 115 compared with adjacent ZG, allowing the differentiation from APA harboring other mutations 116 or without mutations identified [20,25].

117 More than 20 mutations have been identified in CACNA1D (encoding the voltage118 dependent L-type calcium channel subunit alpha-1D, Cav1.3) [26]. The Cav1.3 calcium 119 channel consists of 4 repeat domains, each one consisting of six transmembrane segments, 120 with a membrane-associated loop between S5 and S6 [27-29]. Mutations occurring in 121 CACNA1D are gain of function mutations that lead to a decrease in the threshold of the 122 voltage-dependent activation or impaired channel inactivation, which is followed by increased 
123 intracellular calcium concentrations and thereby an induction of aldosterone biosynthesis $124[28,29]$.

$125 \quad A T P 1 A 1$ and ATP2B3 are members of the P-type family of ATPases and are composed of 12610 transmembrane domains (M1 - M10) with intracellular N- and C-termini. ATP1Al codes 127 for the $\mathrm{Na}(+) / \mathrm{K}(+)$ ATPase alpha-1 subunit. Mutations in this pump lead to a loss of its 128 activity and affinity to $\mathrm{K}^{+}$and to an inward proton or sodium leak, which has been proposed 129 to induce aldosterone production through cell membrane depolarization and increased calcium 130 influx [28,30]. Nevertheless, transient transfection of 2 of the described ATP1A1 mutations in 131 the adrenocortical cell line H295R did not result in modifications of basal cytosolic calcium 132 levels, and barely increased potassium-stimulated calcium concentrations, in spite of 133 depolarizing the cells and stimulating aldosterone biosynthesis. In these cells, Stindl et al 134 found that there was an increased intracellular acidification, which was suggested to regulate 135 CYP11B2 biosynthesis [31].

ATP2B3 codes for the plasma membrane calcium-transporting ATPase 3 (PMCA3).

137 Mutations of PMCA3 are found in the transmembrane domain M4 and result in the deletion 138 of different amino acids in the region between Leu422 and Leu433. One mutation in 139 particular, p.Leu425_Val426del, leads to reduced calcium export which is due to the loss of 140 the physiological pump functions, and an increased intracellular calcium signaling due to the 141 depolarization-activated $\mathrm{Ca}^{2+}$ channels [32]. Recently, a second mechanism explaining 142 aldosterone production due to $A T P 2 B 3$ mutations was suggested. $A T P 2 B 3$ mutations induce 143 an increase in calcium influx by the opening of depolarization-activated calcium channels and 144 by a possible calcium leak through the mutated PMCA3 [32].

$145 C A C N A 1 H$ encoding the pore-forming $\alpha 1$ subunit of the T-type voltage-dependent calcium 146 channel Cav3.2 has been recently shown to be involved in familial forms of PA in some cases 147 associated with developmental disorders [33,34]. In addition, it was also described as 
germline mutation in a patient with APA [34]. This channel consists of a single polypeptide

149 chain of four homologous domains (I-IV), each one containing six transmembrane spans (S1-

150 S6) and cytoplasmic C- and N- Termini. Mutant Cav3.2 channels show significant changes in

151 their electrophysiological properties, specifically a shift in activation towards more negative

152 voltages and modifications of their inactivation properties. Consequently, the channels are

153 activated at less depolarized voltages leading to activation of calcium signaling and

154 autonomous aldosterone production $[33,34]$. A germline $C A C N A 1 H$ variant was identified in

155

156

157

158

159

160

161

162

163

164

165

166

167

168

169

170

171

172 one patient with APA without somatic mutations and improvement after adrenalectomy $[33,34]$. This case suggest that $C A C N A 1 H$ might be a susceptibility gene for different types of PA, including APA.

The Wnt/ $\beta$-Catenin signaling pathway has been shown to play an important role in the development of the adrenal cortex and in aldosterone biosynthesis [35]. This signaling pathway is constitutively active in $\sim 70 \%$ of APA [36]. In unstimulated conditions, $\beta$-catenin is located in the cytosol, and is part of the axin complex along with adenomatous poliposis

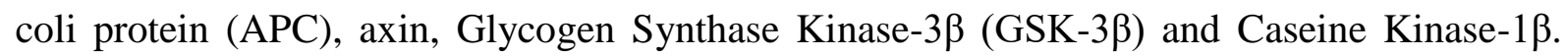
Eventually, $\beta$-catenin in this complex will be phosphorylated resulting in its degradation by the proteasome, and preventing its translocation to the nucleus and the activation of different Wnt target-genes. The activation of the pathway occurs through binding of Wnt ligand to its receptor Frizzled resulting in the inhibition of the phosphorylation of $\beta$-catenin, which dissociates from the axin complex and translocates to the nucleus where it induces the expression of Wnt target genes, most notably the transcription factors T-cell factor $(T C F)$ and lymphocyte enhancer factor $(L E F)$, through its actions as a transcriptional coactivator [35]. Mutations in the CTNNB1 gene, encoding $\beta$-Catenin, have been described in $2-5 \%$ of APA $[17,19]$. The description of somatic CTNN1B mutations associated with higher expression of luteinizing hormone-chorionic gonadotropin receptor (LHCGR) and gonadotropin-releasing 
173 hormone receptor (GNRHR) in APA diagnosed during pregnancy or menopause suggested 174 that pregnancy may reveal an underlying PA [37]. Other studies, however, showed high expression of GNRHR and LHCGR in more than $40 \%$ of APA [38,39], and the presence of CTNNB1 mutations both in females and males $[17,19]$. Further studies are necessary to establish the mechanism of CTNNB1 mutations in the development of APA.

To a much lesser extent, somatic PRKACA (encoding the catalytic $\alpha$ subunit of Protein Kinase A) mutations have been described in APA [18]. Rhayem et al identified somatic mutations of the PRKACA gene in two patients with APA by whole exome sequencing. The mutation p.Leu206Arg, previously identified in CPA [40-42], was found in one patient with PA and Cushing syndrome. The second mutation (p.His88Asp) was identified in a patient without cortisol hypersecretion [18]. This particular mutation was not associated with a gain of function, the mechanism underlying increased PKA signaling and tumorigenesis in cortisol producing adenoma. The role of these mutations on aldosterone secretion and their frequency in APA remains to be established.

CTNNB1 mutations and PRKACA mutations are also identified in cortisol producing adenomas (CPA). Other evidences for an overlap of genetic determinants of aldosterone and cortisol excess have been described, including the cortisol co-secretion observed in a subset of APA, notably those harboring KCNJ5 mutations [43]. The complex mechanisms that would explain how the same mutations could end up in two different hormonal phenotypes remain to be discovered.

\section{Clinical correlates of somatic mutations}

The discovery of clinical or biochemical surrogate markers of somatic mutations in APA could be of benefit for the management of the disease. Different studies described the higher prevalence of somatic KCNJ5 mutations in women and in young patients with APA 
$198[10,16,44]$. KCNJ5 mutations were also associated to higher levels of plasma aldosterone and 199 larger tumors [16], and with higher left ventricular mass index [45]. CACNA1D mutations 200 were associated with smaller APA [10]. More recently, KCNJ5 mutations were described as a

201 predictor of better outcome in young patients with APA [46]. Promising data for the 202 identification of the underlying APA genotype came from a study that analyzed steroid 203 profiles in adrenal and peripheral venous plasma samples from APA patients by liquid 204 chromatography-tandem mass spectrometry [47]. The authors identified a 7-steroid 205 fingerprint in peripheral venous samples allowing to correct classify $92 \%$ of the APA 206 accordingly to genotype. Additionally, specific steroid profiles were associated with KCNJ5 207 mutations, in particular the presence of significantly higher hybrid steroids 18208 hydroxycortisol and 18-oxocortisol. This approach may be translated into clinical care, 209 allowing to identify the APA genotype from peripheral venous plasma samples before surgery. 210 This could be useful for the selection of patients for adrenal vein sampling.

\section{Heterogeneity of APA}

213 In spite of the fact that the relation between aldosterone production and the presence of 214 somatic mutations is well established, the impact of these mutations on nodule/APA 215 formation and cell proliferation is still far from fully understood. APA present a highly 216 pronounced molecular heterogeneity not only on a mutational status but also in terms of 217 aldosterone synthase expression within the same APA. Recent studies showed the presence of 218 different somatic mutations in different aldosterone-producing nodules from the same adrenal $219[48,49]$, suggesting that somatic mutations are independent events occurring in a previously 220 remodeled adrenal cortex. In the same context, Nanba et al described one case of a patient that 221 was diagnosed with PA and Cushing syndrome with double adrenocortical adenomas 222 harboring each a $K C N J 5$ and a PRKACA somatic mutation [50]. Furthermore, in APA 
223 exhibiting heterogeneity of aldosterone synthase expression, APA driver mutations were

224 identified only in positive aldosterone synthase regions [51]. Interestingly, two different 225 mutations were identified in the same APA, lying in two distinct positive aldosterone synthase 226 regions [51]. These findings suggest that somatic mutations are second hits in APA 227 development that emerge from specific mechanisms that remain to be elucidated. Supporting 228 this hypothesis, our group described the occurrence of a germline $A P C$ mutation and a 229 somatic KCNJ5 mutation leading to the development of an APA in a young patient with 230 severe unilateral PA, bilateral macronodular adrenal hyperplasia and Gardner syndrome [52], 231 suggesting a two-hit model for APA development with the APC mutation driving nodule 232 formation and the $K C N J 5$ mutation being responsible for aldosterone hypersecretion.

233 Another theory was suggested by Nishimoto et al, in a study that describes aldosterone 234 producing cell clusters (APCC) in being the origin behind APA development [53]. APCCs are structures of outer morphological ZG cells in contact with the capsule and inner ZF-like cells, staining positive for both CYP11B2 and CYP11B1 [53,54]. They are found in normal adrenal tissue and in adrenals with APA. APCC express important amounts of aldosterone synthase in 238 normal and pathological conditions. In a later study, Nishimoto et al sequenced DNA from 239 APCCs that were collected from normal adrenal glands and identified mutations in APA 240 driver genes in up to $35 \%$ of the collected samples; specifically, mutations in CACNA1D, 241 ATP1A1 and ATP2B3. Interestingly, no mutations in $K C N J 5$ were reported, which is the most 242 frequently mutated gene in APA [55]. The authors suggest that APCCs could represent 243 cellular precursors that could lead to APA with their specific mutations through unknown 244 mechanisms. On the other hand, they propose that APCCs with KCNJ5 could be rarer, or that 245 APCCs that develop KCNJ5 mutations tend to become APAs quite quickly and are hard to be 246 witnessed before the APA development [55]. It was suggested that the sequence of events 247 leading to APA development from an APCC occurs trough the development of structures 
called possible APCC-to-APA translational lesions (pAATL) [56]. pAATL are composed by

249 an outer APCC-like portion and inner micro-APA (mAPA)-like portion. The genetic 250 characterization of pAATL is complex. In one adrenal, a KCNJ5 mutation was identified only

251 in the mAPA-like portion of a pAATL, not observed in the APCC-like portion and was

252 different from the mutation identified in an APA within the same adrenal. This suggests that 253 the APA and the pAATL do not share the same origin and that the KCNJ5 mutation leads to 254 differentiation of the mAPA portion from the APCC. In a second adrenal, both portions of the 255 pAATL carried an ATP1A1 mutation indicating its clonal origin. Although the model 256 whereby APA arise from APCC through pAATL and mAPA is intriguing, further studies are 257 required to better clarify the suite of genomic events involved in this transition.

\section{Conclusion}

The role of each mutation in the regulation of aldosterone production is well studied, while

261 the impact of these mutations on cell proliferation remains to be established. In the future it 262 would be of relevance to distinguish additional biomarkers or the development of techniques 263 that are able to identify somatic mutations in APA. This could be of interest since PA is the 264 most frequent form of secondary hypertension and is curable by the surgical removal of the 265 APA carrying adrenal if recognized early enough. An additional benefit is the possibility of 266 developing new diagnostic and therapeutic approaches. This is particularly the case for the 267 use of macrolides in the detection and treatment of APA with KCNJ5 mutations [57]. A recent 268 work has shown that macrolide antibiotics, including roxithromycin, are potent inhibitors of 269 KCNJ5 channels carrying the most frequent mutations p.Gly151Arg and p.Leu168Arg. Use of 270 clarithromycin in primary cultures from APA showed a significant inhibition of CYP11B2 271 gene expression and aldosterone production [58]. These compounds could therefore be used 
272 to identify patients carrier of APA with KCNJ5 mutations and as targeted treatments in 273 patients who are not candidates for surgery.

274

275

276 


\section{Sources of Funding}

278 This work was funded through institutional support from INSERM and by the Agence 279 Nationale pour la Recherche (ANR Blanc 2011, No.: 11-BSV1 005 03, ANR-13-ISV1-0006280 01), the Fondation pour la Recherche Médicale (DEQ20140329556), the Programme 281 Hospitalier de Recherche Clinique (PHRC grant AOM 06179), and by grants from INSERM 282 and Ministère Délégué à la Recherche et des Nouvelles Technologies. The laboratory of Dr 283 Maria-Christina Zennaro is also partner of the H2020 project ENSAT-HT grant $\mathrm{n}^{\circ} 633983$.

284

\section{Disclosures}

286 The authors have nothing to disclose. 
1. $\quad$ Collaboration, N.C.D.R.F. Worldwide trends in blood pressure from 1975 to 2015: a pooled analysis of 1479 population-based measurement studies with 19.1 million participants. Lancet 2017;389:37-55.

2. Hannemann, A. \& Wallaschofski, H. Prevalence of primary aldosteronism in patient's cohorts and in population-based studies--a review of the current literature. Horm Metab Res 2012;44:157-62.

3. Calhoun, D.A., Nishizaka, M.K., Zaman, M.A., Thakkar, R.B. \& Weissmann, P. Hyperaldosteronism among black and white subjects with resistant hypertension. Hypertension 2002;40:892-6.

4. Douma, S., Petidis, K., Doumas, M., Papaefthimiou, P., Triantafyllou, A., Kartali, N. et al. Prevalence of primary hyperaldosteronism in resistant hypertension: a retrospective observational study. Lancet 2008;371:1921-6.

5. Funder, J.W., Carey, R.M., Mantero, F., Murad, M.H., Reincke, M., Shibata, H. et al. The Management of Primary Aldosteronism: Case Detection, Diagnosis, and Treatment: An Endocrine Society Clinical Practice Guideline. J Clin Endocrinol Metab 2016;101:1889-916.

6. Funder, J.W., Carey, R.M., Fardella, C., Gomez-Sanchez, C.E., Mantero, F., Stowasser, M. et al. Case detection, diagnosis, and treatment of patients with primary aldosteronism: an endocrine society clinical practice guideline. J Clin Endocrinol Metab 2008;93:3266-81.

7. Williams, T.A., Lenders, J.W.M., Mulatero, P., Burrello, J., Rottenkolber, M., Adolf, C. et al. Outcomes after adrenalectomy for unilateral primary aldosteronism: an international consensus on outcome measures and analysis of remission rates in an international cohort. Lancet Diabetes Endocrinol 2017;5:689-699.

8. Mulatero, P., Monticone, S., Bertello, C., Viola, A., Tizzani, D., Iannaccone, A. et al. Long-term cardio- and cerebrovascular events in patients with primary aldosteronism. J Clin Endocrinol Metab 2013;98:4826-33.

9. Spat, A., Hunyady, L. \& Szanda, G. Signaling Interactions in the Adrenal Cortex. Front Endocrinol (Lausanne) 2016;7:17.

10. Fernandes-Rosa, F.L., Williams, T.A., Riester, A., Steichen, O., Beuschlein, F., Boulkroun, S. et al. Genetic spectrum and clinical correlates of somatic mutations in aldosterone-producing adenoma. Hypertension 2014;64:354-61.

11. Hong, A.R., Kim, J.H., Song, Y.S., Lee, K.E., Seo, S.H., Seong, M.W. et al. Genetics of Aldosterone-Producing Adenoma in Korean Patients. PLoS One 2016;11:e0147590.

12. Taguchi, R., Yamada, M., Nakajima, Y., Satoh, T., Hashimoto, K., Shibusawa, N. et al. Expression and mutations of KCNJ5 mRNA in Japanese patients with aldosteroneproducing adenomas. J Clin Endocrinol Metab 2012;97:1311-9.

13. Wang, B., Li, X., Zhang, X., Ma, X., Chen, L., Zhang, Y. et al. Prevalence and characterization of somatic mutations in Chinese aldosterone-producing adenoma patients. Medicine (Baltimore) 2015;94:e708.

14. Wu, V.C., Huang, K.H., Peng, K.Y., Tsai, Y.C., Wu, C.H., Wang, S.M. et al. Prevalence and clinical correlates of somatic mutation in aldosterone producing adenomaTaiwanese population. Sci Rep 2015;5:11396.

15. Zheng, F.F., Zhu, L.M., Nie, A.F., Li, X.Y., Lin, J.R., Zhang, K. et al. Clinical characteristics of somatic mutations in Chinese patients with aldosteroneproducing adenoma. Hypertension 2015;65:622-8. 
16. Lenzini, L., Rossitto, G., Maiolino, G., Letizia, C., Funder, J.W. \& Rossi, G.P. A MetaAnalysis of Somatic KCNJ5 K(+) Channel Mutations In 1636 Patients With an Aldosterone-Producing Adenoma. J Clin Endocrinol Metab 2015;100:E1089-95.

17. Akerstrom, T., Maharjan, R., Sven Willenberg, H., Cupisti, K., Ip, J., Moser, A. et al. Activating mutations in CTNNB1 in aldosterone producing adenomas. Sci Rep 2016;6:19546.

18. Rhayem, Y., Perez-Rivas, L.G., Dietz, A., Bathon, K., Gebhard, C., Riester, A. et al. PRKACA Somatic Mutations Are Rare Findings in Aldosterone-Producing Adenomas. J Clin Endocrinol Metab 2016;101:3010-7.

19. Scholl, U.I., Healy, J.M., Thiel, A., Fonseca, A.L., Brown, T.C., Kunstman, J.W. et al. Novel somatic mutations in primary hyperaldosteronism are related to the clinical, radiological and pathological phenotype. Clin Endocrinol (Oxf) 2015;83:779-89.

20. Boulkroun, S., Golib Dzib, J.F., Samson-Couterie, B., Rosa, F.L., Rickard, A.J., Meatchi, T. et al. KCNJ5 mutations in aldosterone producing adenoma and relationship with adrenal cortex remodeling. Mol Cell Endocrinol 2013;371:2217.

21. Krapivinsky, G., Gordon, E.A., Wickman, K., Velimirovic, B., Krapivinsky, L. \& Clapham, D.E. The G-protein-gated atrial K+ channel IKACh is a heteromultimer of two inwardly rectifying K(+)-channel proteins. Nature 1995;374:135-41.

22. Choi, M., Scholl, U.I., Yue, P., Bjorklund, P., Zhao, B., Nelson-Williams, C. et al. K+ channel mutations in adrenal aldosterone-producing adenomas and hereditary hypertension. Science 2011;331:768-72.

23. Oki, K., Plonczynski, M.W., Luis Lam, M., Gomez-Sanchez, E.P. \& Gomez-Sanchez, C.E. Potassium Channel Mutant KCNJ5 T158A Expression in HAC-15 Cells Increases Aldosterone Synthesis. Endocrinology 2012;153:1774-82.

24. Scholl, U.I., Nelson-Williams, C., Yue, P., Grekin, R., Wyatt, R.J., Dillon, M.J. et al. Hypertension with or without adrenal hyperplasia due to different inherited mutations in the potassium channel KCNJ5. Proc Natl Acad Sci U S A 2012;109:2533-8.

25. Fernandes-Rosa, F.L., Amar, L., Tissier, F., Bertherat, J., Meatchi, T., Zennaro, M.C. et al. Functional histopathological markers of aldosterone producing adenoma and somatic KCNJ5 mutations. Mol Cell Endocrinol 2015;408:220-6.

26. Zennaro, M.C., Boulkroun, S. \& Fernandes-Rosa, F. Genetic Causes of Functional Adrenocortical Adenomas. Endocr Rev 2017;38:516-537.

27. Catterall, W.A. Signaling complexes of voltage-gated sodium and calcium channels. Neurosci Lett 2010;486:107-16.

28. Azizan, E.A., Poulsen, H., Tuluc, P., Zhou, J., Clausen, M.V., Lieb, A. et al. Somatic mutations in ATP1A1 and CACNA1D underlie a common subtype of adrenal hypertension. Nat Genet 2013;45:1055-60.

29. Scholl, U.I., Goh, G., Stolting, G., de Oliveira, R.C., Choi, M., Overton, J.D. et al. Somatic and germline CACNA1D calcium channel mutations in aldosteroneproducing adenomas and primary aldosteronism. Nat Genet 2013;45:1050-4.

30. Beuschlein, F., Boulkroun, S., Osswald, A., Wieland, T., Nielsen, H.N., Lichtenauer, U.D. et al. Somatic mutations in ATP1A1 and ATP2B3 lead to aldosteroneproducing adenomas and secondary hypertension. Nat Genet 2013;45:440-4, $444 \mathrm{e} 1-2$. 
31. Stindl, J., Tauber, P., Sterner, C., Tegtmeier, I., Warth, R. \& Bandulik, S. Pathogenesis of Adrenal Aldosterone-Producing Adenomas Carrying Mutations of the $\mathrm{Na}(+) / \mathrm{K}(+)-A T P a s e$. Endocrinology 2015;156:4582-91.

32. Tauber, P., Aichinger, B., Christ, C., Stindl, J., Rhayem, Y., Beuschlein, F. et al. Cellular Pathophysiology of an Adrenal Adenoma-Associated Mutant of the Plasma Membrane Ca(2+)-ATPase ATP2B3. Endocrinology 2016;157:2489-99.

33. Scholl, U.I., Stolting, G., Nelson-Williams, C., Vichot, A.A., Choi, M., Loring, E. et al. Recurrent gain of function mutation in calcium channel CACNA1H causes earlyonset hypertension with primary aldosteronism. Elife 2015;4:e06315.

34. Daniil, G., Fernandes-Rosa, F.L., Chemin, J., Blesneac, I., Beltrand, J., Polak, M. et al. CACNA1H Mutations Are Associated With Different Forms of Primary Aldosteronism. EBioMedicine 2016;13:225-236.

35. El Wakil, A. \& Lalli, E. The Wnt/beta-catenin pathway in adrenocortical development and cancer. Mol Cell Endocrinol 2011;332:32-7.

36. Berthon, A., Drelon, C., Ragazzon, B., Boulkroun, S., Tissier, F., Amar, L. et al. WNT/beta-catenin signalling is activated in aldosterone-producing adenomas and controls aldosterone production. Hum Mol Genet 2014;23:889-905.

37. Teo, A.E., Garg, S., Shaikh, L.H., Zhou, J., Karet Frankl, F.E., Gurnell, M. et al. Pregnancy, Primary Aldosteronism, and Adrenal CTNNB1 Mutations. N Engl J Med 2015;373:1429-36.

38. Nakamura, Y., Hattangady, N.G., Ye, P., Satoh, F., Morimoto, R., Ito-Saito, T. et al. Aberrant gonadotropin-releasing hormone receptor (GnRHR) expression and its regulation of CYP11B2 expression and aldosterone production in adrenal aldosterone-producing adenoma (APA). Mol Cell Endocrinol 2014;384:102-8.

39. Nicolini, G., Balzan, S., Morelli, L., Iacconi, P., Sabatino, L., Ripoli, A. et al. LH, progesterone, and TSH can stimulate aldosterone in vitro: a study on normal adrenal cortex and aldosterone producing adenoma. Horm Metab Res 2014;46:318-21.

40. Goh, G., Scholl, U.I., Healy, J.M., Choi, M., Prasad, M.L., Nelson-Williams, C. et al. Recurrent activating mutation in PRKACA in cortisol-producing adrenal tumors. Nat Genet 2014;46:613-7.

41. Cao, Y., He, M., Gao, Z., Peng, Y., Li, Y., Li, L. et al. Activating hotspot L205R mutation in PRKACA and adrenal Cushing's syndrome. Science 2014;344:913-7.

42. Beuschlein, F., Fassnacht, M., Assie, G., Calebiro, D., Stratakis, C.A., Osswald, A. et al. Constitutive activation of PKA catalytic subunit in adrenal Cushing's syndrome. N Engl J Med 2014;370:1019-28.

43. Arlt, W., Lang, K., Sitch, A.J., Dietz, A.S., Rhayem, Y., Bancos, I. et al. Steroid metabolome analysis reveals prevalent glucocorticoid excess in primary aldosteronism. JCI Insight 2017;2:

44. Boulkroun, S., Beuschlein, F., Rossi, G.P., Golib-Dzib, J.F., Fischer, E., Amar, L. et al. Prevalence, Clinical, and Molecular Correlates of KCNJ5 Mutations in Primary Aldosteronism. Hypertension 2012;59:592-8.

45. Rossi, G.P., Cesari, M., Letizia, C., Seccia, T.M., Cicala, M.V., Zinnamosca, L. et al. KCNJ5 gene somatic mutations affect cardiac remodelling but do not preclude cure of high blood pressure and regression of left ventricular hypertrophy in primary aldosteronism. J Hypertens 2014;32:1514-21; discussion 1522.

46. Kitamoto, T., Omura, M., Suematsu, S., Saito, J. \& Nishikawa, T. KCNJ5 mutation as a predictor for resolution of hypertension after surgical treatment of aldosterone-producing adenoma. J Hypertens 2018;36:619-627. 
47. Williams, T.A., Peitzsch, M., Dietz, A.S., Dekkers, T., Bidlingmaier, M., Riester, A. et al. Genotype-Specific Steroid Profiles Associated With Aldosterone-Producing Adenomas. Hypertension 2016;67:139-45.

48. Dekkers, T., ter Meer, M., Lenders, J.W., Hermus, A.R., Schultze Kool, L., Langenhuijsen, J.F. et al. Adrenal nodularity and somatic mutations in primary aldosteronism: one node is the culprit? J Clin Endocrinol Metab 2014;99:E134151.

49. Fernandes-Rosa, F.L., Giscos-Douriez, I., Amar, L., Gomez-Sanchez, C.E., Meatchi, T., Boulkroun, S. et al. Different Somatic Mutations in Multinodular Adrenals With Aldosterone-Producing Adenoma. Hypertension 2015;66:1014-22.

50. Nanba, K., Omata, K., Tomlins, S.A., Giordano, T.J., Hammer, G.D., Rainey, W.E. et al. Double adrenocortical adenomas harboring independent KCNJ5 and PRKACA somatic mutations. Eur J Endocrinol 2016;175:K1-6.

51. Nanba, K., Chen, A.X., Omata, K., Vinco, M., Giordano, T.J., Else, T. et al. Molecular Heterogeneity in Aldosterone-Producing Adenomas. J Clin Endocrinol Metab 2016;101:999-1007.

52. Vouillarmet, J., Fernandes-Rosa, F., Graeppi-Dulac, J., Lantelme, P., DecaussinPetrucci, M., Thivolet, C. et al. Aldosterone-Producing Adenoma With a Somatic KCNJ5 Mutation Revealing APC-Dependent Familial Adenomatous Polyposis. J Clin Endocrinol Metab 2016;101:3874-3878.

53. Nishimoto, K., Nakagawa, K., Li, D., Kosaka, T., Oya, M., Mikami, S. et al. Adrenocortical zonation in humans under normal and pathological conditions. J Clin Endocrinol Metab 2010;95:2296-305.

54. Boulkroun, S., Samson-Couterie, B., Dzib, J.F., Lefebvre, H., Louiset, E., Amar, L. et al. Adrenal cortex remodeling and functional zona glomerulosa hyperplasia in primary aldosteronism. Hypertension 2010;56:885-92.

55. Nishimoto, K., Tomlins, S.A., Kuick, R., Cani, A.K., Giordano, T.J., Hovelson, D.H. et al. Aldosterone-stimulating somatic gene mutations are common in normal adrenal glands. Proc Natl Acad Sci U S A 2015;112:E4591-9.

56. Nishimoto, K., Seki, T., Kurihara, I., Yokota, K., Omura, M., Nishikawa, T. et al. Case Report: Nodule Development From Subcapsular Aldosterone-Producing Cell Clusters Causes Hyperaldosteronism. J Clin Endocrinol Metab 2016;101:6-9.

57. Scholl, U.I., Abriola, L., Zhang, C., Reimer, E.N., Plummer, M., Kazmierczak, B.I. et al. Macrolides selectively inhibit mutant KCNJ5 potassium channels that cause aldosterone-producing adenoma. J Clin Invest 2017;127:2739-2750.

58. Caroccia, B., Prisco, S., Seccia, T.M., Piazza, M., Maiolino, G. \& Rossi, G.P. Macrolides Blunt Aldosterone Biosynthesis: A Proof-of-Concept Study in KCNJ5 Mutated Adenoma Cells Ex Vivo. Hypertension 2017;70:1238-1242. 
Figure 1. Regulation of aldosterone biosynthesis in zona glomerulosa cells. (A) In

478 basal conditions, zona glomerulosa cells are in a hyperpolarized state due to the activity of 479 potassium channels at the cell membrane. (B) The binding of AngII to its receptor AT1R or 480 the increase of extracellular $\mathrm{K}^{+}$concentration lead to inhibition of $\mathrm{K}^{+}$currents through TASK 481 and GIRK4 channels, followed by cell membrane depolarization; AngII also inhibits the 482 activity of the $\mathrm{Na}^{+} / \mathrm{K}^{+}$-ATPase pump (ATPlAl) activity. This depolarization leads to the 483 opening of voltage gated calcium channels on the cell membrane increasing $\mathrm{Ca}^{2+}$ 484 concentrations in the cytosol. AngII also induces, through inositol triphosphate (IP3), the 485 release of $\mathrm{Ca}^{2+}$ from the sarco/endoplasmic reticulum. The increased intracellular $\mathrm{Ca}^{2+}$ 486 concentration leads to the activation of the calcium signaling pathway, the major trigger for 487 aldosterone biosynthesis. (C) In pathological conditions, mutations affecting specific ion 488 channels (CACNA1D, CACNA1H, KCNJ5) and ATPases (ATP1A1, ATP2B3) lead to 489 constitutively depolarized ZG cell membrane or directly to increased intracellular $\mathrm{Ca}^{2+}$ 490 concentrations, constitutively activating $\mathrm{Ca}^{2+}$ signaling. The net result is an increased 491 expression of $C Y P 11 B 2$ and an autonomous aldosterone biosynthesis. 


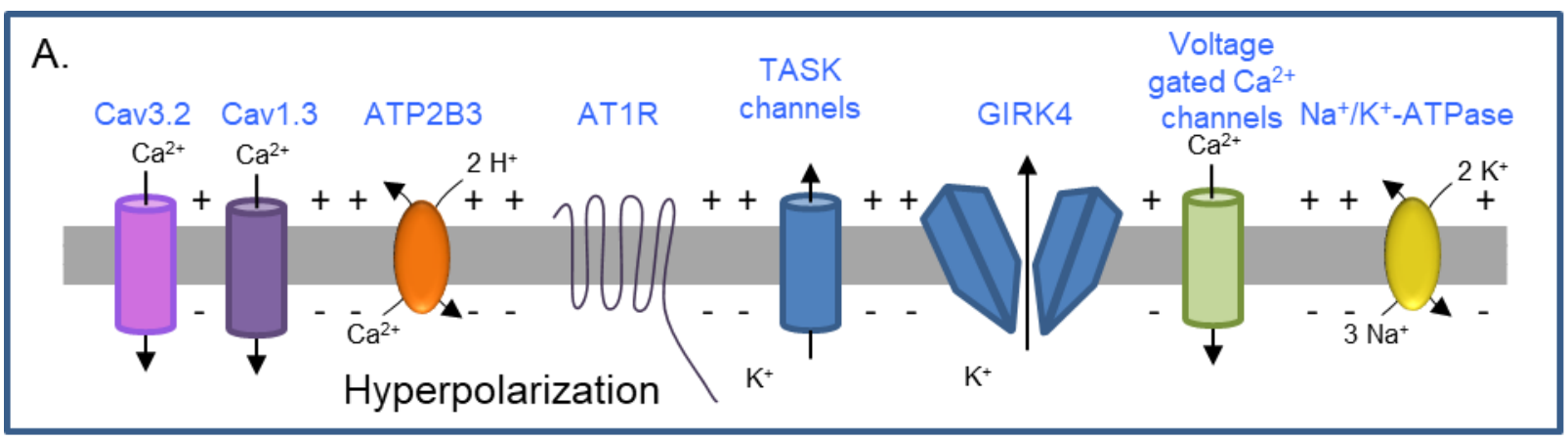

B.

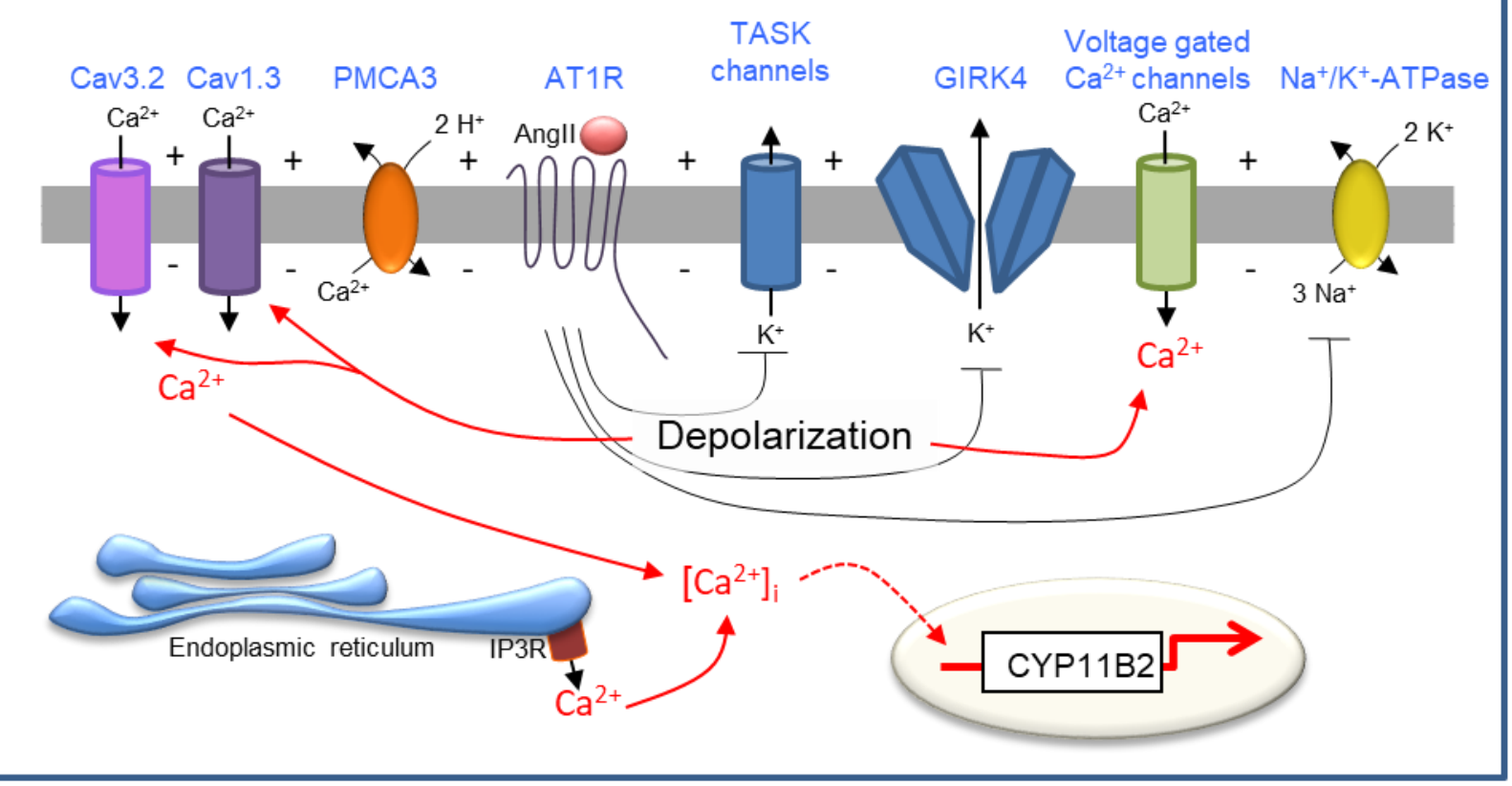

C. 